\title{
Quasi-symmetry and the nature of radial turbulent transport in quasi-poloidal stellarators
}

\author{
J.A. Alcuson ${ }^{1}$, J.M. Reynolds-Barredo, A. Bustos, R. Sanchez and V. Tribaldos \\ Departamento de Física, Universidad Carlos III de Madrid, 28911 Leganés, Madrid, Spain \\ P. Xanthopoulos \\ Max-Planck-Institut für Plasmaphysik, 17491 Greifswald, Germany \\ T. Goerler \\ Max-Planck-Institut für Plasmaphysik, 85748 Garching, Germany \\ D.E. Newman \\ Department of Physics, University of Alaska, Fairbanks, AK 99795, USA
}

(Dated: June 28, 2016)

\begin{abstract}
Quasi-symmetric configurations have a better neoclassical confinement compared to that of standard stellarators. The reduction of the neoclassical viscosity along the direction of quasi-symmetry should facilitate the self-generation of zonal flows and, consequently, the mitigation of turbulent fluctuations and the ensuing radial transport. Therefore, it is expected that quasi-symmetries should also result in better confinement properties regarding radial turbulent transport. In this paper we show that, at least for quasi-poloidal configurations, the influence of quasi-symmetry on radial transport exceeds the expected reduction of fluctuation levels and associated effective transport coefficients, and that the intimate nature of transport itself is affected. In particular, radial turbulent transport becomes increasingly subdiffusive as the degree of quasi-symmetry becomes larger. This behavior is reminiscent of what has been found in tokamaks with strong radially-sheared zonal flows.
\end{abstract}

PACS numbers: 52.25.Fi, 52.35.Ra, 52.25.Gj, 05.40.-a

\section{INTRODUCTION}

An effective manner to confine neoclassical guiding centre orbits in stellarators is by endowing the confining magnetic field with a hidden symmetry (usually referred to as quasi-symmetry), even if just approximate ${ }^{2,3}$. This is best achieved by expressing first the field in Boozer coordinates ${ }^{4}\left(s, \theta_{B}, \phi_{B}\right)$, so that the guiding center motion depends solely on the field magnitude, $B=|\mathbf{B}|$, and its derivatives ${ }^{5}$. Then, the configuration must be designed so that the harmonic content of $B$ is dominated by some linear combination, $M_{\mathrm{qs}} \theta_{B}-N_{\mathrm{qs}} \phi_{B}$, over as much of the radius as possible. Quasi-poloidal symmetry requires that $M_{\mathrm{qs}}=0$, being $N_{\mathrm{qs}}$ arbitrary. Quasi-axisymmetry (or quasi-toroidicity), that $N_{\mathrm{qs}}=0$, bringing the configuration closer to a tokamak. Finally, quasi-helical symmetry, assumes that $M_{\mathrm{qs}} / N_{\mathrm{qs}}=m_{h} / n_{h}$, for some prescribed pair of integers. Examples are provided, respectively, by the $\operatorname{HSX}^{8}$ quasi-helical $\left(m_{h}=1, n_{h}=4\right)$ stellarator currently at operation at the University of Wisconsin, and the QPS $^{6}$ (quasi-poloidal) and $\mathrm{NCSX}^{7}$ (quasiaxisymmetric) projects in the US, that were regretfully cancelled. Experimental results from HSX have already provided evidence supporting an improved neoclassical confinement ${ }^{9}$ and a reduced neoclassical viscosity along the quasi-symmetry direction ${ }^{10}$. One would also expect that, thanks to the reduced neoclassical viscosities, zonal flows able to regulate turbulent fluctuations should be more easily self-generated in configurations with quasisymmetries $^{11}$. However, HSX plasmas are probably too low- $\beta$ to explore this question experimentally. The available numerical evidence does however suggest that this might be the case. Reduced ion turbulent conductivities have been reported for recent gyrokinetic simulations of electrostatic ion-temperature-gradient (ITG) turbulence for numerical equilibria with quasi-helical (i.e., HSX ${ }^{12}$ ) and quasi-axisymmetric $\left(\mathrm{NCSX}^{13}\right)$ geometries. The high computational cost of these runs made that the spatial domains be not global, but limited to the neighborhood of a single magnetic surface, though.

In principle, one would expect that turbulence in quasipoloidal symmetric configurations should be even more sensitive since strong radially-sheared poloidal zonal flows affect radial fluxes the most in configurations with a large safety factor. The investigation of whether this is indeed the case is one of the objectives of this paper. Our studies go however beyond the mere quantification of the reduction of the effective ion conductivity that could be achieved by introducing quasi-poloidal symmetry. The main objective is to find out whether quasisymmetries do also alter the nature of radial transport itself at a deeper level. This investigation is inspired by some recent studies that found a fundamental change in the nature of radial turbulent transport across strong, radially-sheared zonal flows in tokamaks ${ }^{14-16}$. In particular, these previous works reported that radial transport became endowed with significant subdiffusive features as the average radial shear of the poloidal angular flow velocity was increased. At the same time, the statistics of the radial velocity fluctuations became strongly non- 
Gaussian, exhibiting algebraic tails that pointed to the establishment of some kind of non-local correlations. As a result, it was then concluded that a phenomenological description of radial transport in terms of effective coefficients was probably insufficient to capture the transport dynamics in tokamaks with the presence of sufficiently strong sheared-flows, and that other models would probably have to be looked for in order to maintain good predictive capabilities $^{17-21}$.

The present paper thus investigates whether radial turbulent transport could be affected in a similar fashion by the presence of local quasi-symmetries in the confining magnetic field. This question is explored by characterizing the features of the transport of a population of tracked particles as they are advected by the electrostatic ITG turbulence computed by the GENE gyrokinetic code 22,23 in a quasi-symmetric magnetic configuration. The computational domain used in our studies is different from the popular flux-tube geometry used extensively in tokamaks in that it considers the radial neighborhood of a full magnetic surface ${ }^{24,25}$, labeled by its Boozer radial coordinate $s=s_{0}$, instead of just around a given magnetic field line. The degree of quasi-poloidal symmetry at this reference surface is quantified in terms of the quasi-poloidal symmetry ratio,

$$
\sigma_{\mathrm{qp}}\left(s_{0}\right)=\frac{\sum_{n=-N}^{N}\left|B_{0, n}\left(s_{0}\right)\right|}{\sum_{m=0}^{M} \sum_{n=-N}^{N}\left|B_{m, n}\left(s_{0}\right)\right|} \leq 1,
$$

where $(m, n)$ represent the Fourier harmonics associated to the Boozer angles, $\theta_{B}, \phi_{B}$, and $M$ and $N$ are the limiting harmonics included in the representation [The poloidal index $m$ is never negative because we will be always assuming stellarator symmetry $\left.{ }^{2}\right]$. Perfect quasi-poloidal symmetry requires $\sigma_{q p}=1$. For real quasipoloidal configurations, however, $\sigma_{q p}$ is typically a decreasing function of $s$. We have taken advantage of this fact by carrying out all the simulations used in this paper at several radial locations within a single magnetic configuration. In this way, we can achieve a controlled variation of other features of the configuration and still explore a wide range of values of the quasi-poloidal symmetry ratio.

The rest of the paper is organized as follows. First, the configuration used and the simulations carried out for this study are described in Sec. II. The fundamentals of TRACER, the new code that has been developed to advance the tracked particles in the electrostatic potential fluctuations that GENE provides, are also discussed. Sec. III briefly reviews the theoretical aspects of fractional transport theory that are needed to understand the diagnostics used to characterize the nature of transport later in the paper. The application of these diagnostics to the different GENE simulations is described in Sec. IV. The interpretation of the results are discussed at length

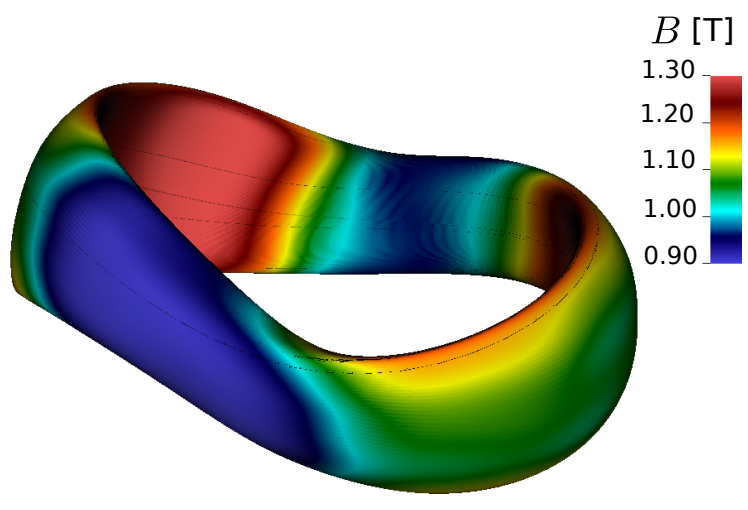

FIG. 1. Quasi-poloidal configuration under study shown in real space. It has $N_{\mathrm{fp}}=2$ field periods. The variation of $B=|\mathbf{B}|$ on the last magnetic surface is shown as a color plot. The quasi-poloidal symmetry can be appreciated from the fact that the contours of constant $B$ tend to be aligned predominantly in the poloidal direction [Although strictly speaking, quasi-poloidal symmetry however requires that $B=B\left(s, \phi_{B}\right)$ instead of $B(s, \phi)$, being $\phi$ the toroidal geometrical angle.].

in Sec. V. Finally, conclusions are presented in Sec. VI.

\section{CONFIGURATION UNDER STUDY}

The quasi-poloidal equilibrium configuration chosen for this investigation belongs to a set of cases explored during the design phase of the QPS project ${ }^{6}$ (see Fig. 1). It is a configuration with two periods (i.e., $N_{\mathrm{fp}}=2$ ), aspect ratio $R_{0} / a=2.6\left(R_{0}\right.$ and $a$ are, respectively, the major and minor radius), $\beta=\left\langle 2 \mu_{0} p / B^{2}\right\rangle \sim 2.5 \%$, toroidal current $I \sim 40 \mathrm{kA}$ and magnetic field on axis, $B_{0} \simeq 0.9$ T. Regarding its magnetic harmonic content, it includes poloidal modes from $m=0$ up to $m=M$, with $M=8$. The toroidal modes are $|n| \leq N$, with $N=7$. The safety factor (defined as $q(s):=\mathrm{d} \Phi / \mathrm{d} \Psi$, with $\Phi(s)$ and $\Psi(s)$ re-

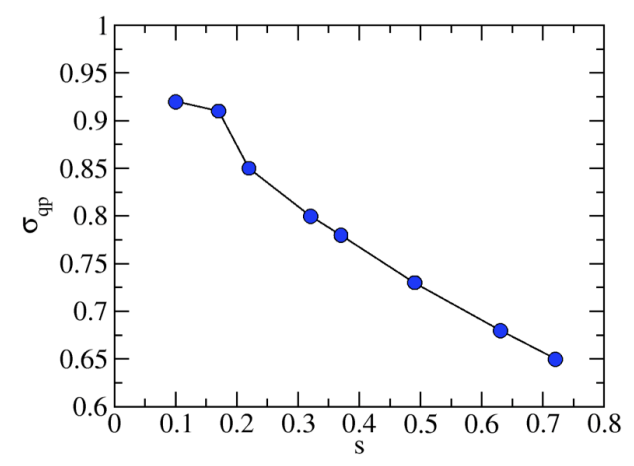

FIG. 2. Quasi-poloidal symmetry ratio $\sigma_{\mathrm{qp}}$ as a function of the Boozer radial coordinate $s \in[0,1]$ for the configuration under study. The surfaces that have been used for the GenE simulations are marked. 
spectively being the toroidal and poloidal magnetic fluxes at the magnetic surface $s$ ) decreases smoothly with radius, from $q(0) \simeq 8.0$ to $q(a) \simeq 4.5$. The quasi-poloidal nature of the symmetry is appreciated in the contour levels of B (see Fig. 1), that are predominantly aligned with the poloidal direction (even when, strictly speaking, the quasi-symmetric alignment is with $\theta_{B}$, not the geometrical poloidal angle). The quasi-poloidal symmetry ratio (Eq. 1) decreases steadily from slightly above 0.9 , close to the axis, to about $\sim 0.6$ as we move closer to the edge (see Fig. 2).

We have carried out nonlinear ITG gyrokinetic simulations with the GENE code $\mathrm{e}^{22,23}$ at eight different radial locations of this configuration. These positions, marked in Fig. 2 with solid circles, provide a significant variation of the quasi-poloidal symmetry ratio. At each radial position, GENE solves the nonlinear gyrokinetic equations ${ }^{26}$ using an Eulerian $\delta f$ approach on a fixed grid in $5 \mathrm{D}$ phase space $\left(x, y, z, v_{\|}, \mu\right)$. As a result, both the nonMaxwellian part of the gyroaveraged ion distribution $(\delta f)$ and the electrostatic potential $\tilde{\phi}$ can be followed in time (see Fig. 3). The spatial domain considered in our simulations is one full annulus centered at a reference surface $s_{0}$ [Here, $s$ is the magnetic toroidal flux normalized to its edge value.]. A special field-aligned coordinate system is used in which $y=C\left(s_{0}\right)\left(q(s) \theta_{B}-\phi_{B}\right)$ labels the magnetic field line, where $C\left(s_{0}\right)=a \sqrt{s_{0}} / q\left(s_{0}\right)$ is a normalization constant. The coordinate $z=\theta_{B}$ runs along the magnetic field line and $x=\sqrt{s}-\sqrt{s_{0}}$ is a normalized radial coordinate. Coordinates respectively span the domains $x \in\left[-L_{x} / 2, L_{x} / 2\right], y \in\left[0, L_{y}\right]$ and $z \in[-\pi, \pi]$. Although originally a flux-tube tokamak code, GENE can deal with this type of annular regions but requires that

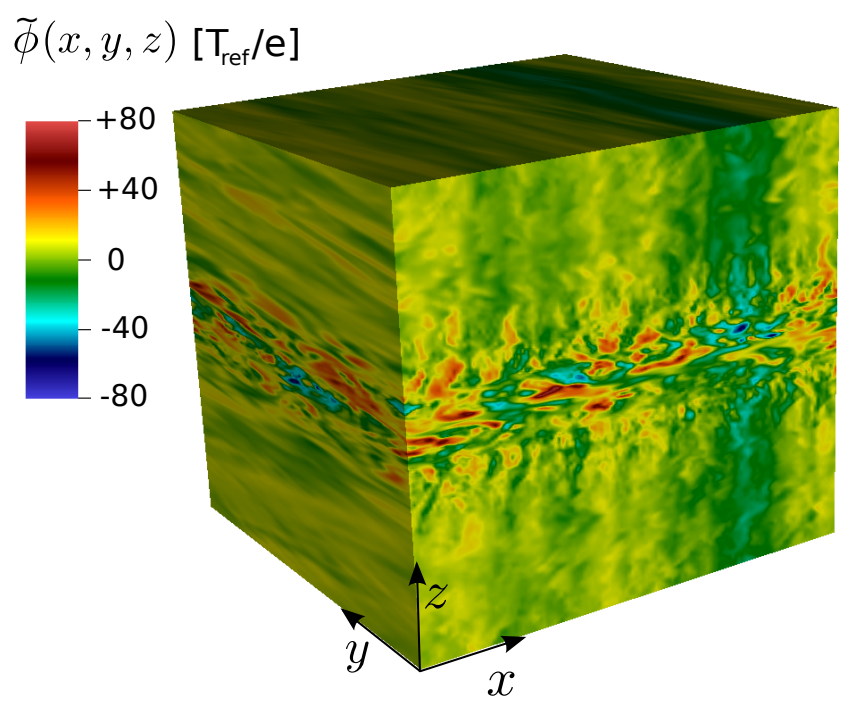

FIG. 3. Example of turbulent electrostatic potential in the field-aligned $(x, y, z)$ coordinates for a selected time well within the saturated region. The cubic view is just used for convenience, since all coordinates are periodic. Data corresponds to the simulation carried out at the $s_{0}=0.49$ surface. another code, GIST ${ }^{27}$, calculates all the geometrical and magnetic information at the magnetic surface $s_{0}$. All equilibrium quantities are assumed to be independent of $x$, but may depend on $y$ and $z$ (in particular, those related to the magnetic field). Periodic boundary conditions in $x$ (and naturally, also in $y$ and $z$ ) are enforced for all perturbed fields. Because of these boundary conditions, $L_{y}$ is not a free parameter, being set to a value that depends on the safety factor at the magnetic surface of interest. The dominant instability in all the simulations performed is the electrostatic ITG mode $\left(a / L_{T_{i}}=4\right.$ and $a / L_{n}=0$ have been used for the ion temperature and density gradients), and adiabatic electrons are assumed. The resolution in $\left(x, y, z, v_{\|}, \mu\right)$ phase space has been set to $126 \times 64 \times 256 \times 32 \times 8$ points in all cases. These numbers were chosen after having completed systematic linear and nonlinear convergence studies, in which we monitored the saturation levels of various physical variables such as ion heat flux and parallel and perpendicular temperatures, among others. The radial size of the computational domain, for the parameter values used, contains roughly $L_{x} \simeq 140 \rho_{s}$, with $\rho_{s}=c_{s} / \Omega_{i}$, being $c_{s}=\sqrt{2 T_{e} / m}$ the sound velocity, and $\Omega_{i}=e B / m$ the ion cyclotron frequency. Accordingly, the often used parameter $\rho^{*}=\rho_{s} / a=0.004$. Finally, all simulations have been advanced in time for several hundreds of Lagrangian turbulent decorrelation times, as will be discussed later. Runs must be at least this long to ensure that the features extracted from the transport studies are meaningful.

The techniques that we have used to characterize the nature of transport (see Sec. III) require knowledge of the trajectories of individual particles as they are advected by the turbulence. Advecting particles within modern Vlasov gyrokinetic codes is not a trivial task due to their large computational cost and their sophisticated but fragile parallel optimization ${ }^{28}$. As a result, the most straightforward way to carry out this type of studies -namely, to include the tracked particle evolution within the normal Vlasov-Poisson time-stepping- is not very practical. It would require a major overhaul of the GK code in order to maintain its internal balance for optimal parallelization and performance. In addition, every time that a different tracked particle initialization is needed, the whole GK simulation would have to be rerun, thus incurring in a huge waste of computational resources. For all these reasons, we have developed our own parallel code, TRACER, to carry out the advection of as many particles as desired for as long as needed as a post-process. TRACER uses GENE's standard output to gather all the relevant information needed to complete this task including, but not restricted to, the metric tensor and the jacobian (i.e., geometry), the (time-varying) electrostatic potential (see Fig. 3), the magnetic field and its gradients. TRACER interpolates all these fields in space and in time using either B-splines or linear interpolation. When massless particles are considered (i.e., tracer particles), each trajectory is calculated by integrating the 
equation of motion (see Fig. 4),

$$
\dot{\mathbf{r}}=\mathbf{v}_{\mathrm{E} \times \mathrm{B}}+v_{\|} \frac{\mathbf{B}}{B}=-\frac{\nabla \tilde{\phi} \times \mathbf{B}}{B^{2}}+v_{\|} \frac{\mathbf{B}}{B},
$$

using an explicit 4-th order Runge-Kutta (RK4) scheme. Regarding parallel motion, all tracers are initialized with a value of $v_{\|}$drawn from a Maxwellian distribution at the equilibrium ion temperature that is maintained constant throughout the motion.

TRACER also offers the possibility of tracking trajectories of particles with mass and charge. In that case, perpendicular magnetic drifts are included in the equation of motion:

$$
\dot{\mathbf{r}}=\mathbf{v}_{\mathrm{E} \times \mathrm{B}}+\mathbf{v}_{\text {Magn. drift }}+v_{\|} \frac{\mathbf{B}}{B},
$$

defined by the usual expression,

$$
\mathbf{v}_{\text {Magn. drift }}=\frac{m v^{2}}{2 q B^{3}}\left(1+\frac{v_{\|}^{2}}{v^{2}}\right) \mathbf{B} \times \nabla B .
$$

Here, $m$ and $q$ are respectively the mass and charge of the particle. The parallel velocity is updated in the usual way, assuming conservation of the magnetic moment $\mu$ :

$$
\dot{v}_{\|}=-\frac{\mu}{m} \frac{\mathbf{B} \cdot \nabla B}{B}, \quad \dot{\mu}=0,
$$

thus including a magnetic mirror effect.

Tracked particles, either with mass or massless, can be initially distributed in space in many different ways. The choice must be made depending on the diagnostic used (see Sec. III). For instance, when using the Lagrangian diagnostic technique ${ }^{21}$, it is more convenient to initialize them uniformly throughout the computational domain. To estimate radial propagators ${ }^{20}$, on the other hand, it is preferrable to locate them initially at $x=0$, with a uniform distribution along the $y$ and $z$ directions. It must be noted, however, that a much larger number of particles $\left(\sim 10^{4}-10^{6}\right)$ is often required to get sufficiently good statistics for propagators, compared with the Lagrangian technique $^{17}$. This is due to the fact that the relevant information in this context must be retrieved from the tails of the propagators, that correspond to much smaller probabilities.

\section{DIAGNOSTICS TO CHARACTERIZE FRACTIONAL TRANSPORT}

The main diagnostic that we will use to characterize the nature of transport along the radial (i.e., $x$ ) direction is the analysis of the statistical and correlation properties of the radial component of the Lagrangian velocity ${ }^{17,21}$. This analysis is one of several contrasted techniques that can be used to characterize the nature of transport in turbulent (and other types of) systems. It only requires following in time a sufficiently large population of tracers as they are advected by the background turbulence.

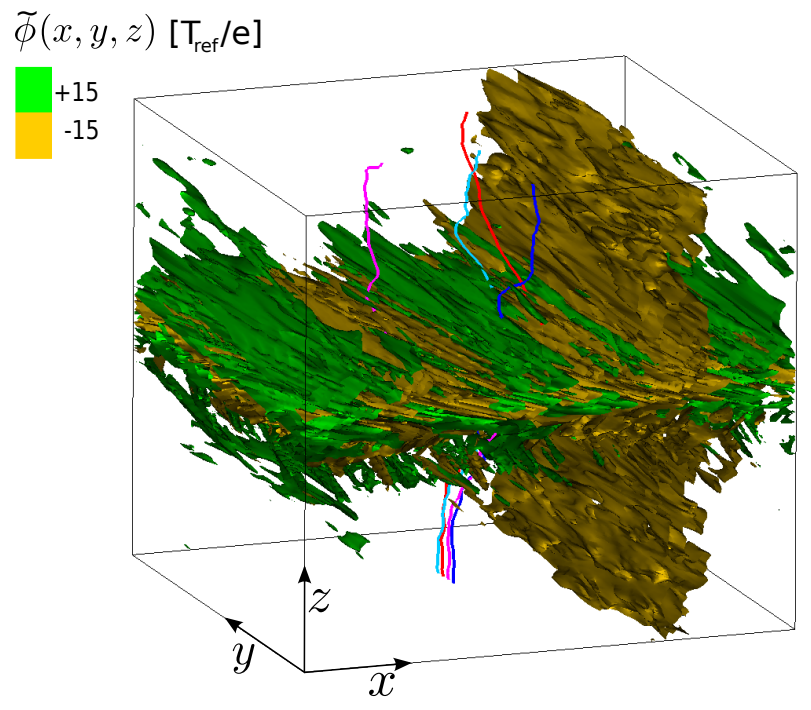

FIG. 4. Example of several massless tracer trajectories obtained by integrating Eq. 2. Although the motion takes place mostly along $z\left(v_{\|}>0\right.$ has been used for those included in the figure), the direction of interest is along $x$. The trajectories are superimposed on (a snapshot of) two selected equipotential surfaces for illustration purposes (In reality, trajectories are integrated using the time-evolving electrostatic potential, not a frozen one). The apparent vertical structure, roughly $m=n=0$, corresponds to a region of zonal flow.

The basis of the technique relies on the comparison of the properties of the motion of a tracked particle with those of fractional Lévy motion (fLm) ${ }^{29,30}$. fLm is a stochastic model that generalizes the popular Langevin equation (LE) from which classical diffusion is often derived. As will be remembered, the Langevin equation gives the position of a single particle as:

$$
x(t)=x_{0}+\int_{0}^{t} \mathrm{~d} t^{\prime} \xi_{2}\left(t^{\prime}\right),
$$

where $\xi_{2}(t)$ is a Gaussian, uncorrelated noise with a correlation function given by $\left\langle\xi_{2}(t) \xi_{2}\left(t^{\prime}\right)\right\rangle=D \delta\left(t-t^{\prime}\right)$. The connection of the Langevin equation with diffusion can be established, for example, by computing the propagator of Eq. 6. That is, the probability of finding the particle at any position $x$ at time $t>0$. It is given by,

$$
P^{\mathrm{LE}}\left(x, t \mid x_{0}\right)=\frac{1}{\sqrt{2 \pi D t}} \exp \left(-\frac{\left(x-x_{0}\right)^{2}}{2 D t}\right) .
$$

This Gaussian propagator, with standard variation growing as $\sigma^{\mathrm{LE}}=(D t)^{1 / 2}$, is also the propagator of the classical diffusion equation,

$$
\frac{\partial n}{\partial t}=D \frac{\partial^{2} n}{\partial x^{2}}
$$

In a real physical system, the role of noise would be played by the (turbulent) Lagrangian velocity of the particle, whose correlation function is often modeled as,

$$
\left\langle v(t) v\left(t^{\prime}\right)\right\rangle \sim v_{c}^{2} \exp \left(-\frac{\left|t-t^{\prime}\right|}{\tau_{c}}\right) .
$$


With this choice, the long-term, long-distance limit of transport is also well described by classical diffusion with $D \sim v_{c}^{2} \tau_{c}$. Therefore, one can conclude that the longterm dominance of diffusive transport is ultimately related to the existence of finite characteristic scales associated to the transport process. Namely, $v_{c}$ and $\tau_{c}$. The velocity $v_{c}$ is related to the (square root of the) variance of the Lagrangian velocitiy; $\tau_{c}$ determines for how long memory is maintained (in the velocity) as the particle advances along its (Lagrangian) trajectory.

There are however situations in which such finite transport scales may be absent in a system. Eq. 6 is not appropriate to model the overall transport dynamics in those cases. A more suitable generalization, able to deal with these cases, is provided by the stochastic equation ${ }^{30}$

$$
x(t)=x_{0}+\frac{1}{\Gamma\left(H-\frac{1}{\alpha}+1\right)} \int_{0}^{t} \mathrm{~d} t^{\prime}\left(t-t^{\prime}\right)^{H-\frac{1}{\alpha}} \xi_{\alpha}\left(t^{\prime}\right),
$$

where $\Gamma(x)$ the Euler's gamma function. Here, $\xi_{\alpha}(t)$ is noise distributed according to an uncorrelated, symmetric Lévy distribution with a tail exponent $\alpha \in(0,2]$. For $\alpha<2$, these distributions have a fat power-law tail, $L_{\alpha}(x) \propto|x|^{-(1+\alpha)}$, and lack a finite variance (in fact, all moments of order $\alpha$ or larger are infinite $\left.{ }^{29}\right)$. Clearly, $\xi_{\alpha}$ will no longer be a surrogate of the Lagrangian velocity. The latter is obtained by differentiating (with care) Eq. 10. The exponent $H \in(0, \max (1,1 / \alpha)]$, on the other hand, is referred to as the Hurst exponent. Its range of possible values is limited to ensure that the propagator of Eq. 10 remains well-behaved ${ }^{29}$.

Eq. 10 contains several famous stochastic models. For instance, the usual Langevin equation is recovered whenever $\alpha=2$ and $H=1 / 2$. If $\alpha=2$ but $H$ is not prescribed, Eq. 10 reduces to the famous fractal Brownian motion (fBm). The propagator is still Gaussian but with a standard deviation that grows in time like ${ }^{31}$ :

$$
\sigma^{\mathrm{fBm}} \propto \frac{(D t)^{H}}{(2 H)^{1 / 2} \Gamma\left(H+\frac{1}{2}\right)} .
$$

The $\mathrm{fBm}$ propagator scales diffusively only if $H=1 / 2$. Otherwise, $\mathrm{fBm}$ transport is either subdiffusive $(H<0.5)$ or superdiffusive $(H>0.5)$. One can also study the properties of $\mathrm{fBm}$ transport from the ordered sequence of its (Lagrangian) velocities (obtained by differentiation of Eq. 10). It turns out that ${ }^{32}$, although the variance of the pdf of these velocities (that sets the velocity scale, $v_{c}$, as we discussed earlier) is still defined, a finite timescale (i.e., $\tau_{c}$ ) no longer exists if $H \neq 1 / 2$. This means that memory is being maintained for infinitely long times along the Lagrangian trajectory, a consequence of the power-law kernel in Eq. 10. As a result, the long-term, long-distance limit of transport no longer corresponds to classical diffusion.

In the case $\alpha \neq 2$, the propagator of Eq. 10 takes the form of a symmetric Lévy law ${ }^{33}$ :

$$
P^{\mathrm{fLm}}\left(x, t \mid x_{0}\right)=t^{-H} L_{\alpha, \sigma^{\mathrm{fLm}}}\left(\frac{x-x_{0}}{t^{H}}\right)
$$

with scale factor $\sigma^{\mathrm{fLm}}$ defined as

$$
\sigma^{\mathrm{fLm}}=\sigma_{\xi_{\alpha}}\left[(\alpha H)^{1 / \alpha} \Gamma\left(H+1-\frac{1}{\alpha}\right)\right]^{-1},
$$

being $\sigma_{\xi_{\alpha}}$ the scale factor of the noise Lévy distribution. This type of motion is known as fractional Levy motion $(\mathrm{fLm})^{30}$. Since all moments of order $\alpha$ or larger are now infinite, a characteristic length for transport is no longer present. However, any moment of order $p<\alpha$ is still finite, scaling as $\left\langle\left(x-x_{0}\right)^{p}\right\rangle \propto t^{p H}$. Following fBm conventions, the scaling $H=1 / 2$ is still referred to as a diffusive scaling (although dynamics are neither Markovian nor Gaussian). Subdiffusion is used for $H<0.5$, superdiffusion, for $H>0.5$. In the case of fLm, neither a finite $v_{c}$ nor a finite $\tau_{c}$ can be defined for its Lagrangian velocities. The former, because velocities are distributed according to a similar Lévy law statistics with the same tail-index $\alpha$, thus lacking a finite variance. The latter, because memory is again maintained for infinitely long times along the Lagrangian trajectory if $H \neq 1 / \alpha$ due to the kernel in Eq. 10, as in $\mathrm{fBm}$.

A way to characterize the nature of transport in a system is to compare its characteristics with those of fLm. That is, one simply needs to estimate the exponents $\alpha$ and $H$ that define fLm. One can do this by characterizing the statistics and the correlation of the (component of interest of the) Lagrangian velocities ${ }^{21}$. Also, one can estimate the transport propagator along the direction of interest $^{20}$. In this paper, we will mostly rely on the first
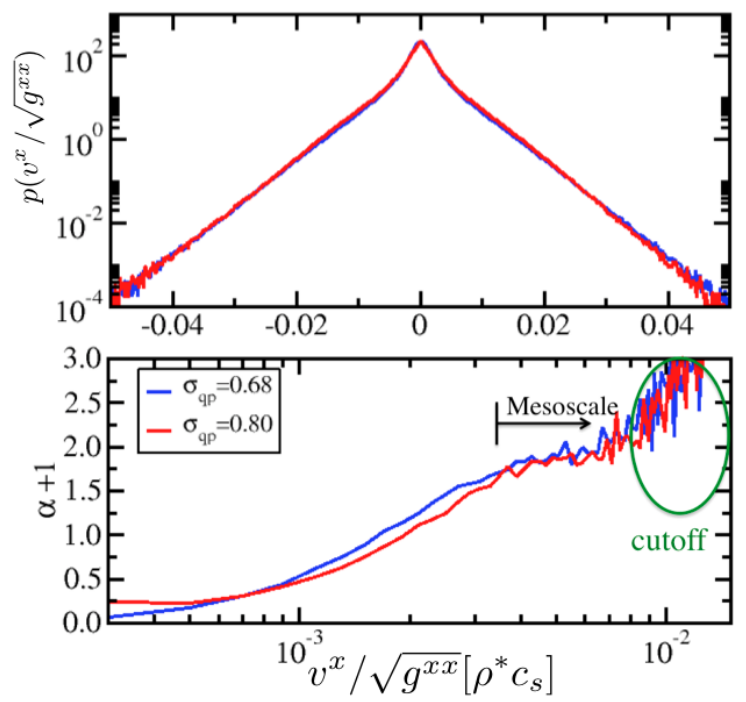

FIG. 5. Above: pdfs of the Lagrangian radial velocities (in log-linear) obtained using ten thousand tracer particles; the blue curve corresponds to tracers advanced in the turbulence calculated around the surface $s_{0}=0.32$, where the quasipoloidal symmtery ratio is $\sigma_{q p}=0.80$; the red one, to tracers advanced around the surface $s_{0}=0.63$, where $\sigma_{q p}=0.68$. Below: instantaneous tail exponent (see Eq. 14) for the two pdfs. The start of the mesoscale range is marked with a vertical line and an arrow. 
approach. A collection of tracked particles, uniformly initizalized throughout the computational box, will be advanced in the presence of the electrostatic turbulence, and their radial Lagrangian velocities recorded. These velocities correspond to $v^{x} / \sqrt{g^{x x}}$ in GENE internal coordinates, since $v^{x}$ is a radial contravariant component and must be divided by the length of the proper contravariant basis vector, $\left|\mathbf{e}^{\mathbf{x}}\right|=\sqrt{g^{x x}}$ (because $\mathbf{e}^{\mathbf{x}}$ points in the radial direction). The exponent $\alpha$ is then estimated from the tail-index of the pdf of the velocity component along the $x$ direction. Or, in other words, by quantifying whether the pdf of these velocities decays (or not) as a power law $p(v) \sim v^{-(1+\alpha)}$ for large $|v|$. The procedure is illustrated in Fig. 5 for massless tracers that have been advanced in the turbulence computed in the neighbourhood of two different magnetic surfaces of the QPS configuration. The flat regions in the lower frame, that shows the local tail exponent, defined as,

$$
\alpha(v)+1:=-(v / p) \mathrm{d} p / \mathrm{d} v,
$$

correspond to the mesorange where a meaningful value for $\alpha$ can be estimated. As seen in the figure, a cutoff appears for larger values of $v$ whose origin is probably related to the limited resolution of the runs (see Sec. V).

To estimate $H$, on the other hand, we use a simple variation of the popular rescaled-range (or R/S) method, introduced by H. E. Hurst in the 50s to quantify memory in Guassian-distributed time series ${ }^{34}$. Assuming a time series $V_{k}, k=1,2, \cdots N$, Hurst's prescription required the computation of the rescaled range:

$$
[R / S](\tau):=\frac{\max _{1 \leq k \leq \tau} W(k, \tau)-\min _{1 \leq k \leq \tau} W(k, \tau)}{\sqrt{\left\langle V^{2}\right\rangle_{\tau}-\langle V\rangle_{\tau}^{2}}},
$$

with

$$
W(k, \tau):=\sum_{i=1}^{k} V_{i}-k\langle V\rangle_{\tau} .
$$

Here, $\langle\cdot\rangle_{\tau}$ represents the temporal average up to time $\tau$. When the signal resembles $\mathrm{fBm}$, then $[R / S] \sim \tau^{H}$, with $H$ being the Hurst exponent. The prescription must however be slightly modified ${ }^{21}$ to deal with fLm, due to the divergent nature of its variance. In those cases, the denominator of the rescaled-range is replaced by the $1 / s$ th power of a moment of order $s>0$, with $s<\alpha$. It is useful to introduce the instantaneous Hurst exponent,

$$
H(\tau):=-\frac{\tau}{[R / S](\tau)} \cdot \frac{\mathrm{d}[R / S]}{\mathrm{d} \tau}(\tau),
$$

that is used to determine the range of scales where $H$ is well defined. They will appear as flat regions in the instantaneous Hurst exponent.

The procedure is illustrated in Fig. 6, that shows the results of performing the $\mathrm{R} / \mathrm{S}$ analysis on the time series of the Lagrangian radial velocity of two tracers advanced
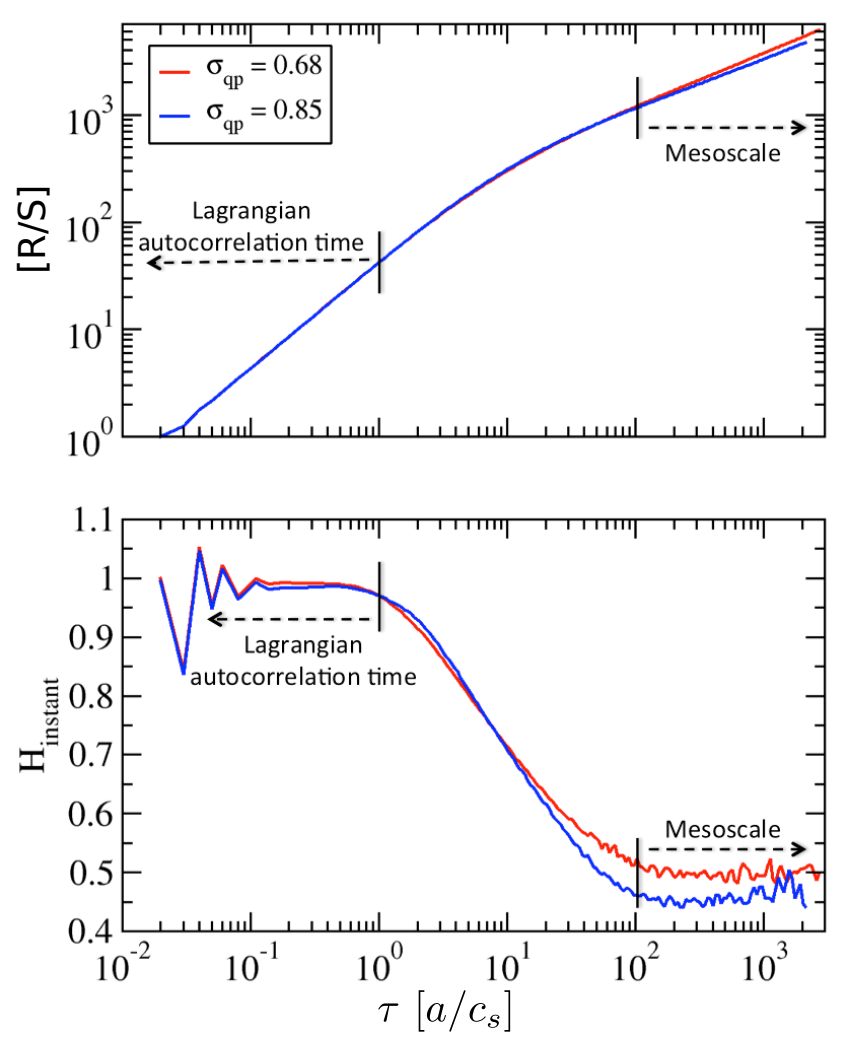

FIG. 6. Above: rescaled range (i.e., $[R / S]$ ) as a function of time delay for two time series of radial Lagrangian velocities of tracers advanced by TRACER; the black one comes from a tracer advanced in the turbulence computed by GENE around $s_{0}=0.22$ where the quasi-poloidal symmetry ratio is $\sigma_{q p}=$ 0.85 ; the red one, from a tracer advanced around $s_{0}=0.63$, where $\sigma_{q p}=0.68$. Below: instantaneous Hurst exponent (Eq. 17) for the same two series. The auto-correlation and mesoscale ranges are marked with vertical lines and arrows.

by TRACER for two different Gene simulations. The shape of the $[R / S]$ function shown in the upper frame is rather typical, with a first range at the lowest time lags in which $[R / S] \sim \tau$ that gives a rough estimate of the Lagrangian auto-correlation time (for the cases shown, it is roughly $\tau_{L} \sim(1-2) a / c_{s}$. At longer scales, about $\tau>10^{2} a / c_{s}$, is where the mesorange dynamics appear in the form of a second power-law range. It is the exponent in this second region that corresponds to the Hurst exponent. The figure shows two distinct cases, one for a tracer advanced in the turbulence calculated at $s_{0}=0.63$ $\left(\sigma_{\mathbf{q p}}=0.68\right)$ in which $[R / S] \sim \tau^{0.49}$, almost diffusive, and a second one advanced at $s_{0}=0.22\left(\sigma_{\mathbf{q p}}=0.85\right)$ where $[R / S] \sim \tau^{0.45}$, that is distinctly subdiffusive.

In the next section we will discuss the results of applying these techniques on tracked particles advanced in the turbulent fields computed at the various surfaces of reference shown in Fig. 2. By monitoring changes in $\alpha$ and/or $H$ as the quasi-poloidal symmetry ratio varies, we will be able to detect any fundamental change responsible for pushing transport dynamics away from the standard 

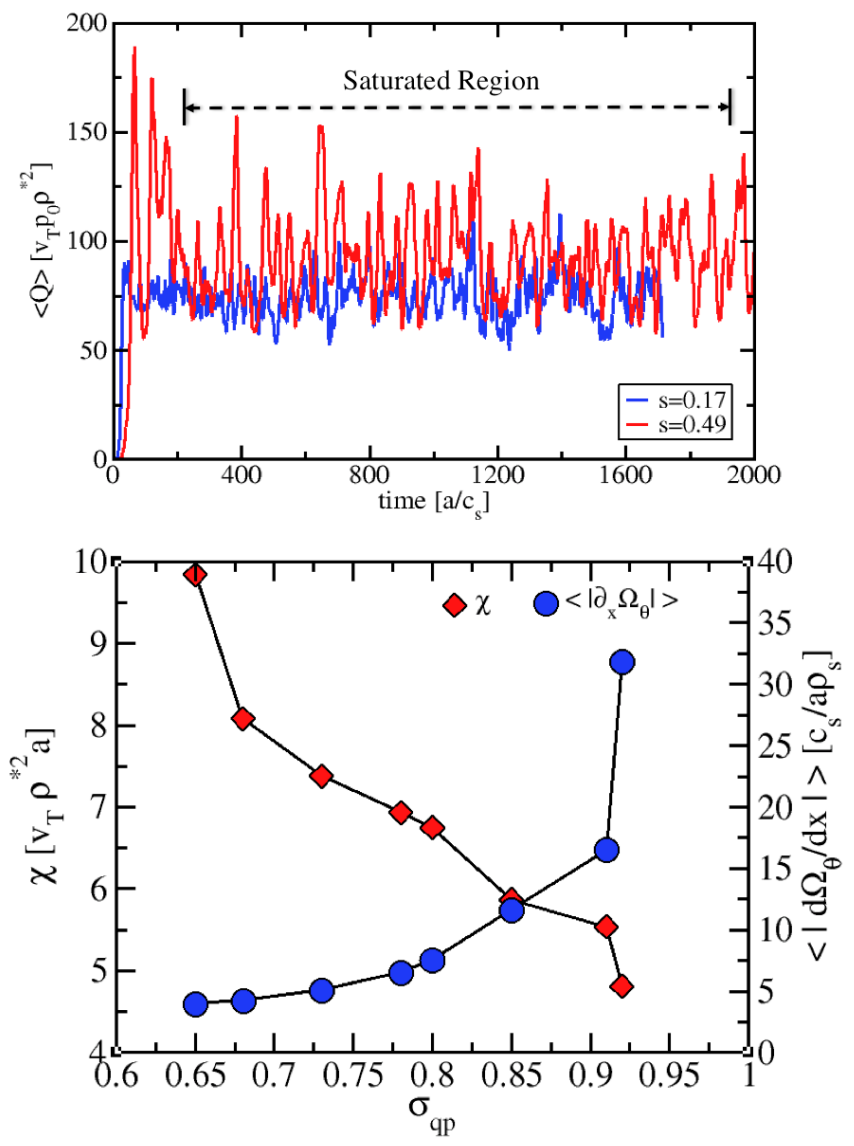

FIG. 7. Above: Time traces of the ion heat flux for two of the simulations examined. Bounded by vertical lines is the range over which tracers are advanced, already within the saturation region. Below: Effective heat conductivity (left, in red) and shear flow strength (right, in blue) as a function of the quasi-poloidal symmetry ratio. We use, as figure-of-merit to quantify the sheared flow strength, the surface average of $\left\langle\left|\mathrm{d} \Omega_{\theta} / \mathrm{d} x\right|\right\rangle$ (see description in text).

framework of classical diffusion.

\section{SIMULATION RESULTS}

ITG nonlinear GK simulations have been carried out for the eight different radial locations of the QPS equilibrium marked in Fig. 2. The range of values of the quasipoloidal symmetry ratio examined extends from 0.65 , at the outermost surface, to 0.92 , almost at the magnetic axis (see also Table. I).

\section{A. Heat fluxes}

The first result worth mentioning from these runs relative to radial transport does not require any tracked particles. It is that, consistently with previous nonlinear simulations for other quasi-symmetries (i.e., quasi- helical $^{12}$ and quasi-axisymmetric ${ }^{13}$ ), the effective ion heat conductivity, computed as the average:

$$
\chi_{i, \text { eff }}=\left\langle\frac{-q^{i}}{\mathrm{~d} T_{i} / \mathrm{d} x}\right\rangle_{\text {volume }},
$$

(being $q^{i}$ the outward ion heat flux), becomes smaller (for the same equilibrium gradient values) as the quasipoloidal symmetry ratio $\sigma_{\mathrm{qp}}$ increases. This is shown in the lower frame of Fig. 7 , where the reduction in $\chi_{i \text {,eff }}$ is also shown to be positively correlated with the capability of self-generated, radially sheared poloidal flows to act on and suppress the turbulence. We have chosen to characterize this capability in terms of the figure-of-merit $\left\langle\left|\mathrm{d} \Omega_{\theta} / \mathrm{d} x\right|\right\rangle_{\text {volume }}$, with the angular velocity $\Omega_{\theta}$ given by:

$$
\Omega_{\theta}=\frac{\left(v_{z}+C\left(s_{0}\right) q_{0} v_{y}\right) / \sqrt{g}}{\left(g_{z z}+C^{2}\left(s_{0}\right) q_{0}^{2} g_{y y}+2 C\left(s_{0}\right) q_{0} g_{y z}\right)},
$$

where $q_{0}=q\left(s_{0}\right)$ is the safety factor at the magnetic surface $s=s_{0}, v_{z}$ and $v_{y}$ are the covariant components of the $\mathbf{E} \times \mathbf{B}$ velocity, and $g_{z z}, g_{y y}$ and $g_{y z}$ are the proper covariant metric elements. $\Omega_{\theta}$ is related to the part of the poloidal angular turbulent velocity that is tangent to the magnetic surface $s=s_{0}$.

\section{B. Massless tracer motion}

We turn next to the analysis of the motion of massless tracers. Thousands of massless tracer particles have been advanced in each case, for periods of time of the order of $\left(10^{3}-10^{4}\right) a / c_{s}$, more than three decades longer than the local Lagrangian decorrelation time (see Fig. 6), and well

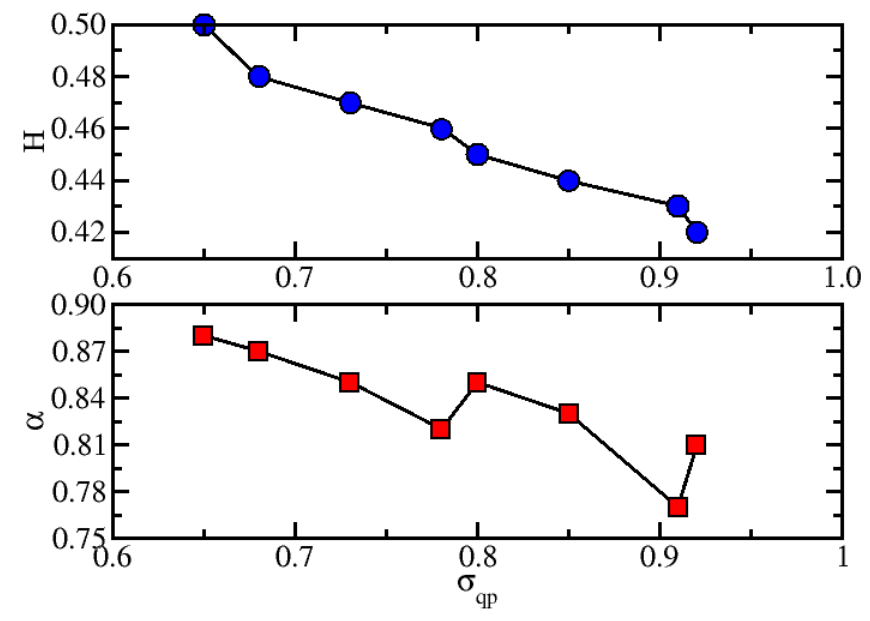

FIG. 8. Values of the exponents $H$ (above) and $\alpha$ (below) obtained for the radial velocity time series as a function of the value of the quasi-poloidal symmetry ratio. 


\begin{tabular}{|c|cccc|}
\hline$s_{0}$ & $\sigma_{\mathrm{qp}}$ & $\left\langle\left|\mathrm{d} \Omega_{\theta} / \mathrm{d} x\right|\right\rangle_{\text {volume }}$ & $H$ & $\alpha$ \\
\hline \hline 0.10 & 0.92 & 31.87 & 0.42 & 0.81 \\
\hline 0.17 & 0.91 & 16.52 & 0.43 & 0.77 \\
\hline 0.22 & 0.85 & 11.65 & 0.44 & 0.83 \\
\hline 0.32 & 0.80 & 7.59 & 0.45 & 0.85 \\
\hline 0.37 & 0.78 & 6.55 & 0.46 & 0.82 \\
\hline 0.49 & 0.73 & 5.12 & 0.47 & 0.85 \\
\hline 0.63 & 0.68 & 4.27 & 0.48 & 0.87 \\
\hline 0.72 & 0.65 & 4.02 & 0.50 & 0.88 \\
\hline \hline
\end{tabular}

TABLE I. List of values of the exponents $H$ and $\alpha$ obtained from the simulations at the neighbourhood of the magnetic surface labeled as $s_{0}$.

deep within the nonlinearly saturated phase. These long periods are essential for the analysis, as we mentioned earlier, since it is the long-term transport features that we are interested in. Following the methodology described in Sec. III, we have estimated the values of the exponents $\alpha$ and $H$ that characterize radial transport. The exponents are determined for each tracer trajectory, and then averaged over all (usually a few thousand) tracers. The averaged values obtained are shown in the upper frame of Fig. 8 as a function of the quasy-poloidal symmetry ratio at each magnetic surface (they have also been collected in Table I). The results show that $H<0.5$ except at the last outermost surface, and that this value is well established for more than a decade starting at $\tau>10^{2} a / c_{s}$ (see Fig.6, lower frame). This result suggests that longterm, subdiffusive radial heat transport is indeed taking place for $s<0.6$, instead of the type of reduced diffusive transport that one might have naively expected. This is an important finding. Furthermore, the value of the exponent $H$ consistently decreases as the value of the quasi-poloidal symmetry ratio, $\sigma_{\mathrm{qp}}$, is increased (see Fig. 5, upper frame). Consequently, transport inherits a more strongly subdiffusive character as the center of the configuration is approached, and becomes less so as we move outwards, until it eventually scales diffusively (in these runs, for $s \geq 0.6-0.7$ ).

Secondly, we observe that the exponent $\alpha$ is significantly non-Gaussian, with $\alpha \sim 0.7-0.9$. In contrast to the case of $H$, the exponent $\alpha$ does not decrease monotously with the value of the quasi-poloidal symmetry ratio (see Fig. 8, lower frame), although there is a globally decreasing trend. That is, it consistently becomes less Gaussian as $H$ becomes more subdiffusive. In this case, however, the region of self-similarity that appears in the velocity pdf, from which $\alpha$ is determined, reaches a cutoff rather quickly at all positions (as illustrated in Fig. 5) that makes the proper determination of $\alpha$ difficult.

In order to gain additional confidence on the obtained values of the exponents $\alpha$ and $H$, we have also estimated them by means of another independent method: radial propagators. Ten thousand massless tracers were initially located at $x=0$ (uniformly distributed in $y$ and $z$ ) and then advected by the turbulence at each surface. The evolution of this population gives an estimate of the ef-
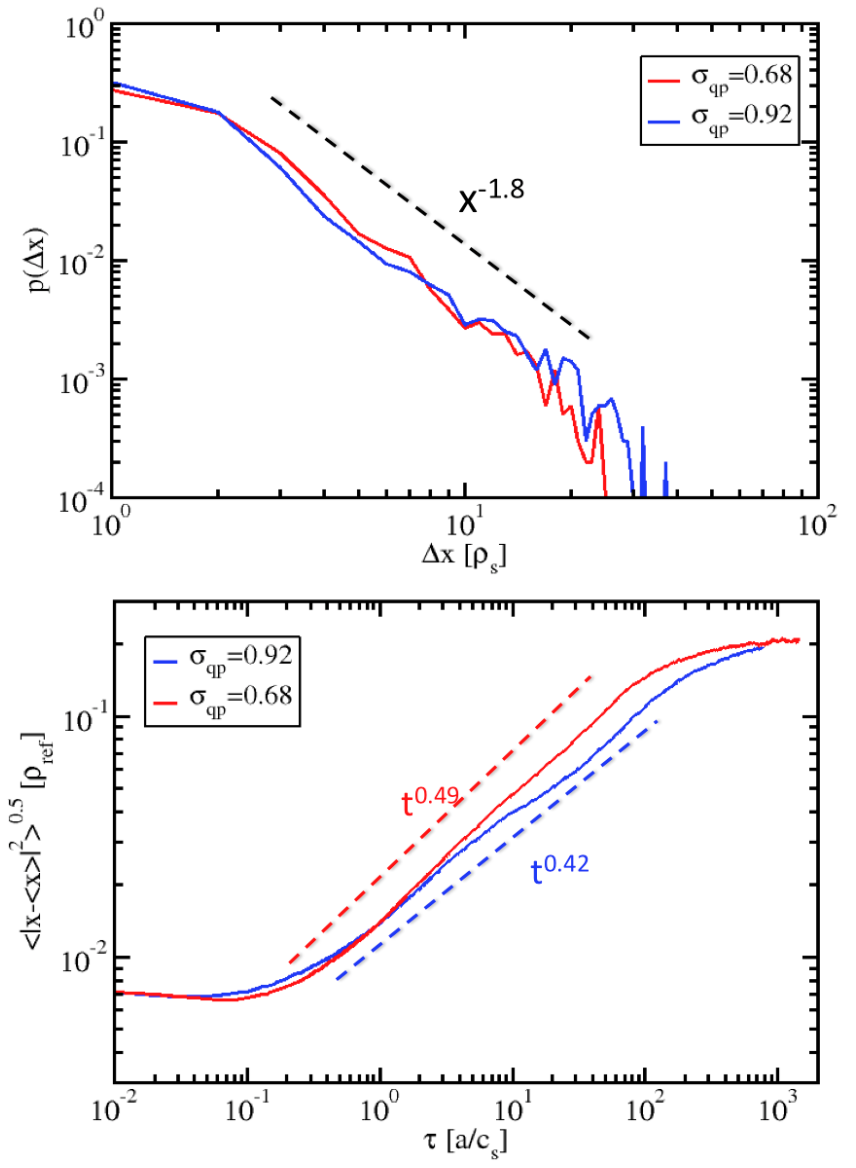

FIG. 9. Positive tail of the estimated radial propagator (in log-log scale, above) and growth with time of its first moment (below) calculated in the neighbourhood of the magnetic surfaces located at $s_{0}=0.10$ and $s_{0}=0.63$. The propagator tail should scale as $P(\Delta x) \sim \Delta x^{-(1+\alpha)}$, from which $\alpha$ is inferred. The moment used should scale as $\tau^{H}$, on the other hand.

fective radial propagator, as discussed in Sec. III. The procedure for the determination of the exponents from the propagators is illustrated in Fig. 9. The upper frame shows, in a log-log scale, the tail of the propagator after a time of the order of $10 a / c_{s}$ for two different locations, one at surface $s_{0}=0.10$, with $\sigma_{\mathrm{qp}}=0.92$, the other at $s_{0}=0.63$, with $\sigma_{\mathrm{qp}}=0.68$. The tails of all fLm propagators scale as $P(\Delta x) \sim \Delta x^{-(1+\alpha)}$. Therefore, a value of $\alpha \sim 0.8$ is inferred, that seems to be a bit steeper for the less quasi-symmetric location, confirming what was already found with the Lagrangian method. The exponent $H$, on the other hand, is estimated from the growth with time of the moments of propagator. This is illustrated in the lower frame of Fig. 9. A value of $H \sim 0.42$ for the inner surface at $s_{0}=0.10$, that has the largest degree of quasi-symmetry, and $H \sim 0.49$ for $s_{0}=0.63$. Again, these values are consistent with what was previously found using the Lagrangian method. 


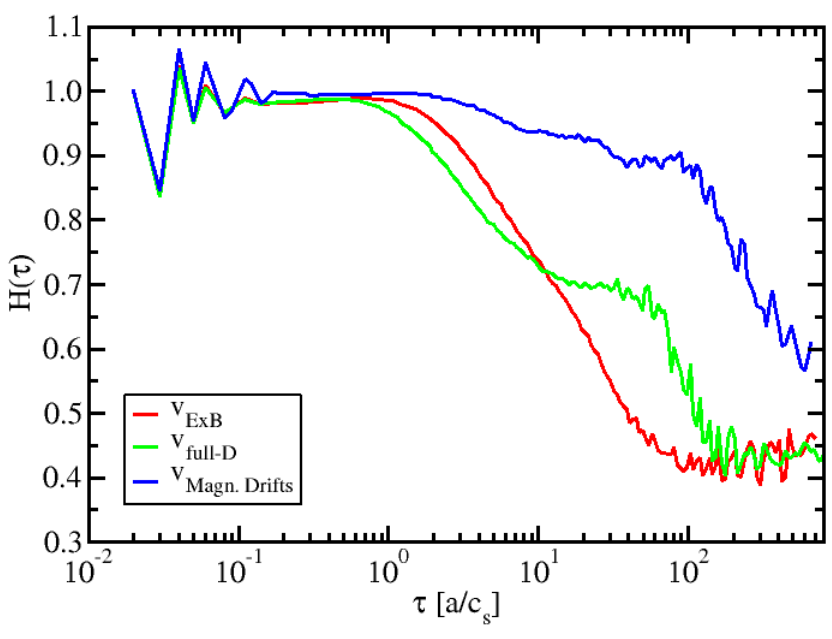

FIG. 10. Instantaneous value of the Hurst exponent $H$ for massless tracers (in red) and thermal ions (in blue) as they are advected by the ITG turbulence in the neighbourhood of the $s_{0}=0.10$ magnetic surface.

\section{Thermal ion motion}

Finally, we have also studied whether the values of the exponents $H$ and $\alpha$ change significantly if the tracked particles considered had the mass, charge and kinetic energy of plasma thermal ions. In this case, their equation of motion includes all magnetic drifts and mirror effects (see Eq. 3). The Lagrangian analysis shows, in this case, that the estimated values for the exponent $\alpha$ barely change. However, things become more interesting with respect to the $H$ exponent. The situation is illustrated in Fig. 10. It compares, for the most subdiffusive simulation (the one carried out in the neighbourhood of $s_{0}=0.10$, with $\sigma_{\mathrm{qp}}=0.92$ ), the instantaneous value of $H$ obtained for thermal ions (in green) with that of massless tracers (in red). We have also plotted the result in the case in which the turbulent $\mathbf{E} \times \mathbf{B}$ drift is not included (in blue). The important thing to notice is that the longterm behaviour of the exponent (for $\tau>10^{2} a / c_{s}$ ) seems to be very similar for thermal ions and tracers, remaining subdiffusive. However, there is a new intermediate range of scales for the thermal ions $\left(10<\tau<10^{2} a / c_{s}\right)$ when a clear superdiffusive plateau becomes apparent, that is absent for massless tracers. A similar trend is observed at all radial locations. We will explain the physics for this new scaling region in the next section.

\section{DISCUSSION}

The numerical results obtained in this study - and described in the previous section - suggest that, although a reduction of the effective ion heat conductivity is indeed observed as the degree of quasi-poloidal symmetry is increased, this fact in itself does not provide the complete picture of what is happening with the radial turbulent transport. Radial transport appears to inherit a subdiffusive character (i.e., $H<0.5$ ), that becomes stronger as the degree of quasi-symmetry becomes larger. The physical mechanism behind this trend seems to be strongly correlated with the increase in the shearing capability of the self-generated turbulent zonal flow in the poloidal direction that is enabled by the reduced neoclassical viscosity associated to the higher degree of quasi-symmetry. This connection can be made more apparent by plotting the obtained values for the exponents in terms of the figure-of-merit that we use to characterize the shearing capability of the zonal flow, $\left\langle\left|\mathrm{d} \Omega_{\theta} / \mathrm{d} x\right|\right\rangle_{\text {volume }}$ (see Fig. 11).

This behaviour is very reminiscent of how radial transport was found to behave in recent gyrokinetic simulations of ITG tokamak turbulence that had strong radially-sheared poloidal flows ${ }^{14,16}$. In fact, most of the available numerical evidence points to the same physical mechanisms as the responsible ones for the subdiffusive radial transport observed here. The physical mechanism for this process can be understood as follows. Any large positive [negative] value of $\left\langle\left|\mathrm{d} \Omega_{\theta} / \mathrm{d} x\right|\right\rangle_{\text {volume }}$, whose generation via the Reynolds stresses is facilitated by the reduced neoclassical viscosity that a larger quasi-poloidal symmetry brings, causes a growth in extension and intensity of those regions with positive [negative] vorticity together and a suppresion of those with negative [positive] vorticity ${ }^{16}$. This process takes place in addition to the concomittant stretching along $y$ and the shortening along $x$ of any region of localized vorticity (along $z$ ) driven by the sheared rotation. The vorticity landscape

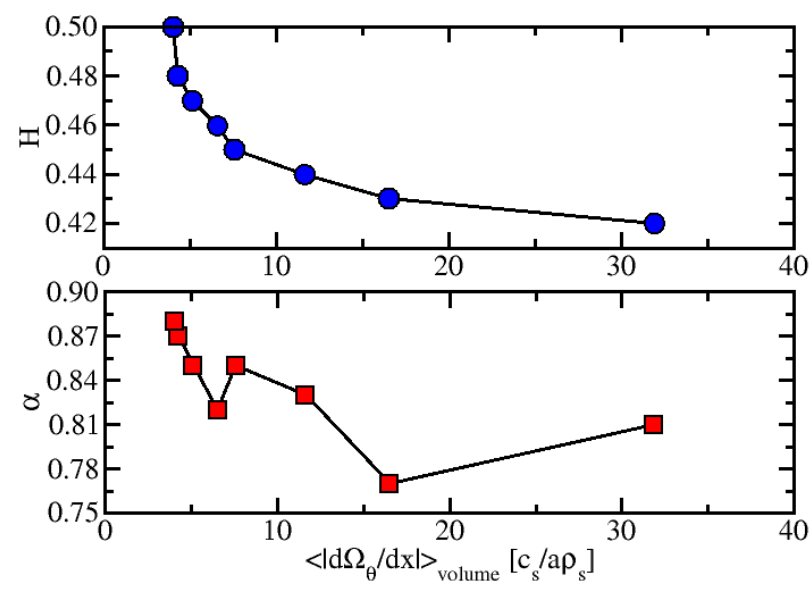

FIG. 11. Values of the exponents $H$ (above) and $\alpha$ (below) obtained for the radial velocity time series as a function of the figure-of-merit characterizing the strength of the shear in the poloidal zonal flow, $\left\langle\left|\mathrm{d} \Omega_{\theta} / \mathrm{d} x\right|\right\rangle_{\text {volume }}$. 
that results from these processes makes it more probable for any radial motion to reverses its direction often, instead of moving steady, which automatically leads to the observed subdiffusion along $x$. This behaviour is insensitive to the sign of the shear, caring only about the shear absolute strength. This is what we observe here. More interestingly, tokamak simulations proved that the establishment of subdiffusion via this process does not require that the zonal flow be self-generated by the turbulence ${ }^{14}$. Transport would also become subdiffusive in the presence of externally driven flows, which suggests an interesting avenue to transport control via external biasing.

Regarding the non-Gaussian tails $(\alpha<2)$ found in our simulations, their meaning is not so clear. In previous tokamak GK simulations the values of the tail exponents found were much larger $(\alpha \sim 1.4-1.5)$ and increased towards the Gaussian value (i.e., $\alpha \sim 2$ ) as the zonal flow shear strength decreased and transport became more diffusive $^{16}$. This is not what we have found in the current GENE simulations for a quasi-poloidal geometry. Instead, $\alpha \sim 0.8-0.9$, although it does seem to increase as the value of the quasi-poloidal symmetry ratio is reduced. In the tokamak GK simulations it was also found that the mechanism responsible for subdiffusion was very different from that causing the lack of Gaussianity. The latter was related to the establishment of a predator-prey cycle between potential and flow shear local fluctuations that translated into avalanche-like radial propagations whose reflection was the appearence of power-law tails. It is not clear at this time if this mechanism is absent or suppressed in our quasipoloidal runs. In addition, the tokamak simulations were done with $\mathrm{UCAN}^{35}$, a global, PIC code. Gene is Eulerian and local. At the moment we cannot say whether the much lower values obtained for the exponent $\alpha$ are caused by differences in the dominant physics caused by the stellarator geometry, or just because of factors related to the different numerical implementation used. It is our intention to further investigate this question in the near future.

In regards to the different behavior of the instantaneous $H$ exponent found for massive ions and illustrated in Fig. 10, its explanation can be traced to the spatial distribution of the sign of the radial component of $\mathbf{B} \times \nabla B$ that governs the magnetic drift of the massive ions. As shown in Fig.12, this sign is predominantly positive (i.e., red) for $z \in(0, \pi)$ and negative (blue) for $z \in(-\pi, 0)$. Since $z$ corresponds to the Boozer poloidal angle, this up-down asymmetry pushes massive ions radially outwards in the upper half of the configuration, and radially inwards in the lower half. But since $z$ is also used as the label that runs along any magnetic field line, massive ions feel these effects alternatively as they move along the field lines due to the relatively large safety factor. The process is ballistic in nature and governs the scaling of radial transport in the intermediate range $\left(10-10^{2}\right) a / c_{s}$ as shown in Fig. 10. This is particularly clear in the blue curve in that figure, that only considers magnetic drifts and parallel motion for the massive ions. When

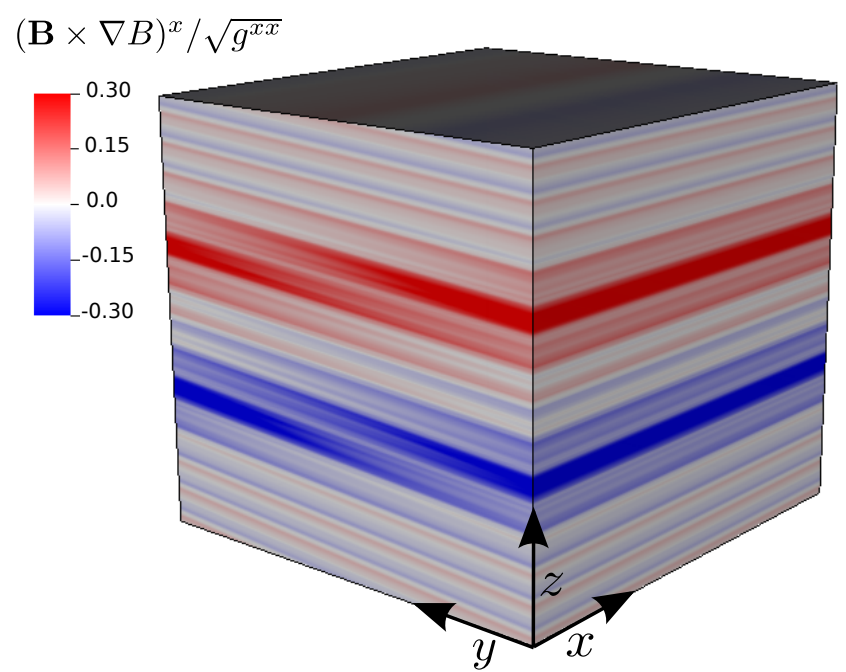

FIG. 12. Variation of the radial component of $\mathbf{B} \times \nabla B$ across the computational volume centered at the magnetic surface $s_{0}=0.10$. Since $z=\theta_{B}$, the poloidal Boozer angle, the plot shows that massive particles are going to be pushed outwards in radius (i.e., to larger $x$ 's) when $z \in(0, \pi)$, and then inwards (to smaller $x$ 's) while $z \in(-\pi, 0)$, as they move along the field line. These ballistic processes are responsible for the superdiffusive behaviour made apparent in Fig. 10.

the contribution to perpendicular motion of the turbulent $\mathbf{E} \times \mathbf{B}$ is also included, as shown by the green curve in Fig. 10, turbulent decorrelation begins to dominates for timescales $\tau>10^{2} a / c_{s}$, beyond which the radial motion of massive ions becomes very similar to that of the massless tracers. That is, it becomes more subdiffusive the larger the quasi-poloidal symmetry ratio is.

\section{CONCLUSIONS}

We have shown that quasi-poloidal symmetry may improve the confinement properties of a three-dimensional magnetic configuration, in regards to radial turbulent transport, in a much deeper sense than through the mere reduction of effective transport coefficients (the ion heat conductivity, in this case). The reduction of the neoclassical viscosity along the direction of quasi-symmetry allows for the development of zonal flows with a stronger radial shear, that is able to alter the vorticity landscape is such a way as to drive transport subdiffusively across the flow (i.e., in the radial direction). The physical mechanisms responsible for this behaviour appear to be analogous to those recently reported for tokamaks. Although some differences are also found, particularly in what refers to the non-Gaussianity of the process, that deserve further study. It is not clear at this time whether this is a fundamental difference or if it is somehow caused by the computational method chosen. This issue will be studied in the future. A last point worth making is that 
subdiffusive transport will not dominate for arbitrarily long timescales, though. Otherwise, it would be impossible to reach a steady state in the present of a constant drive. Therefore, for sufficiently long times, the nature of transport will eventually become diffusive. But those scales can be very large (for the cases examined here, they clearly lie beyond $10^{4} a / c_{s}$, our longest simulation time). Therefore, if the process of interest takes place within the range of scales in which subdiffusion dominates, proper predicitive capabilities will only be maintained if such dynamics are captured properly.

\section{ACKNOWLEDGMENTS}

Research funded in part by the Spanish National Projects ENE2012-33219 and ENE2012-31753. Research supported in part by DOE Office of Science Grant No.
DE-FG02-04ER5741 at University of Alaska. Research carried out in part at the Institüt für Plasmaphysik of the Max-Planck Institüt in Greifswald (Germany), whose hospitality is gratefully acknowledged. Fruitful interactions with members of the ABIGMAP research network, funded by the Spanish National Project MAT2015-69777REDT, is also acknowledged. GENE simulations have been possible thanks in part to a continued grant (Refs. FI-2014-1-0021, FI-2014-2-0026, FI-2014-3-0012 and FI2015-1-0011) to use resources from the MareNostrum supercomputer at BSC (Barcelona, Spain). GENE and TRACER runs have also been carried out in Uranus, a supercomputer cluster located at Universidad Carlos III de Madrid (Spain) funded jointly by EU FEDER funds and by the Spanish Government via the $\mathrm{Na}$ tional Projects UNC313-4E-2361, ENE2009-12213-C0303, ENE2012-33219 and ENE2012-31753.
1 Author to whom correspondence should be addressed. Electronic mail: jalcuson@fis.uc3m.es.

2 AH. Boozer. Rev. Mod. Phys. 761071 (2004).

3 P. Helander. Rep. Prog. Phys. 77087001 (2014).

4 A.H. Boozer. Phys. Fluids 25, 520 (1982).

${ }^{5}$ R.B. White, A.H. Boozer and R. Hay. Phys. Fluids 25, 575 (1982).

6 D.A. Spong, S.P. Hirshman, L.A. Berry, J.F. Lyon, R.H. Fowler, D.J. Strickler, M.J. Cole, B.E. Nelson, D.E. Williamson, A.S. Ware, D. Alban, R. Sanchez, G.Y. Fu, D.A. Monticello, W.H. Miner and P.M. Valanju. Nucl. Fusion 41, 711-716 (2001).

7 M.C. Zarnstorff, L.A. Berry, A. Brooks, E. Fredrickson, G.-Y. Fu, S Hirshman, S. Hudson, L.-P. Ku, E. Lazarus, D. Mikkelsen, D. Monticello, G. H. Neilson, N. Pomphrey, A. Reiman, D. Spong, D. Strickler, A. Boozer, W.A. Cooper, R. Goldston, R. Hatcher, M .Isaev, C. Kessel, J. Lewandowski, J.F. Lyon, P. Merkel, H. Mynick, B. E. Nelson, C. Nuehrenberg, M. Redi, W. Reiersen, P. Rutherford, R. Sanchez, J. Schmidt and R. B. White. Plasma Phys. Control. Fusion 43, A237-A249, (2001).

${ }^{8}$ F. S. B. Anderson, A. F. Almagri, D. T. Anderson, P. G. Mathews, J. N. Talmadge and J. L. Shohet, Fus. Technology 27, 273 (1995).

9 J.M. Canik, D.T. Anderson, F.S.B. Anderson, K.M. Likin, J.N. Talmadge and K. Zhai. Phys. Rev. Lett. 98, 085002 (2007)

10 S.P. Gerhardt, J.N. Talmadge, J.M. Canik and D.T. Anderson. Phys. Rev. Lett. 94, 015002 (2005).

11 P.W. Terry. Rev. Mod. Phys. 72, 109 (2000).

12 W. Guttenfelder, J. Lore, D.T. Anderson, F.S.B. Anderson, J.M. Canik, W. Dorland, K.M. Likin and J.N. Talmadge. Phys. Rev. Lett. 101, 215002 (2008).

13 H. E. Mynick, N. Pomphrey and P. Xanthopoulos Phys. Rev. Lett. 105, 095004 (2010).

14 R. Sanchez, D.E. Newman, J. N. Leboeuf, V.K. Decyk and B.A. Carreras Phys. Rev. Lett. 101205002 (2008).

15 R. Sanchez, D.E. Newman, J.N. Leboeuf, B.A. Carreras and V.K. Decyk Phys. Plasmas 16055905 (2009)
16 R. Sanchez, D.E. Newman, J.N. Leboeuf and V.K. Decyk Plasma Phys. Control. Fusion 53074018 (2011).

17 R. Sanchez and D.E. Newman. Plasma Phys. Contr. Fus. 57, 123002 (2015).

18 B.A. Carreras, V.E. Lynch and G.M. Zaslavski Phys. Plasmas 85096 (2001).

19 B.Ph. Milligen, R. Sanchez and B.A. Carreras Phys. Plasmas 112272 (2004).

20 D. del-Castillo-Negrete, B.A. Carreras and V.E. Lynch Phys. Rev. Lett. 94065003 (2005).

21 J.A. Mier, R. Sanchez, L. Garcia, B.A. Carreras, D.E. Newman Phys. Rev. Lett. 101165001 (2008).

22 F. Jenko, W. Dorland, M. Kotschenreuther and B.N. Rogers Phys. Plasmas 7, 1904 (2000).

${ }^{23}$ T. Görler, X. Lapillonne, S. Brunner, T. Dannert, F. Jenko, F. Merz and D. Told, Journal of Computational Physics 2307053 (2011).

${ }^{24}$ P. Xanthopoulos, H.E. Mynick, P. Helander, Y. Turkin, G.G. plunk, F. Jenko, T. Görler, D. Told, T. Bird and J.H.E. Proll, Phys. Rev. Let. 113, 155001 (2014).

25 P. Xanthopoulos, G.G. Plunk, A. Zocco and P. Helander, Phys. Rev. X 6, 021033 (2016).

26 A.J. Brizard and T.S. Hahm Rev. Mod. Phys. 79, 421 (2007).

27 P. Xanthopoulos, W.A. Cooper, F. Jenko, Y. Turkin, A. Runov and J. Geiger. Phys. Plasmas 16, 082303 (2009).

28 T. Hauff and F. Jenko Phys. Plasmas 13102309 (2006).

29 G. Samorodnitsky and M.S. Taqqu. Stable non-Gaussian processes. Chapman and Hall, New York (1994).

30 N. Laskin, I. Lambadaris, F.C. Harmantzis and M. Devetsikiotis. Comp. Networks 40, 363-375 (2002).

31 B.B. Mandelbrot and J.W. van Ness. SIAM Review 10, 422-437 (1968).

32 R. Sanchez, B.A. Carreras, D.E. Newman, V. Lynch and B.Ph. van Milligen Phys. Rev. E 74016305 (2006).

33 I. Calvo and R. Sanchez, J. Physics A 42, 055003 (2009).

34 H. E. Hurst, Trans. Am. Soc. Civ. Eng. 116, 770 (1951).

35 R.D. Sydora, V.K Decyk and J.M. Dawson, Plasma Phys. Contr. Fus. 38, A281 (1996). 